\title{
Sieve functions in arithmetic bands, II
}

\author{
Giovanni Coppola and Maurizio Laporta
}

\begin{abstract}
An arithmetic function $f$ is called a sieve function of range $Q$ if its Eratosthenes transform $g=f * \mu$ is supported in $[1, Q] \cap \mathbb{N}$, where $g(q) \ll_{\varepsilon} q^{\varepsilon}(\forall \varepsilon>0)$. We continue our study of the distribution of such functions over short arithmetic bands, $n \equiv a r+b(\bmod q)$, with $1 \leq a \leq H=o(N)$ and $r, b \in \mathbb{Z}$ such that g.c.d. $(r, q)=1$. In particular, we discuss the optimality of some results.
\end{abstract}

\section{Introduction.}

Given an arithmetic function $f: \mathbb{N} \rightarrow \mathbb{C}$, for every $N \in \mathbb{N}$ and $\alpha \in \mathbb{R}$ let us set

$$
\widehat{f_{\mathcal{N}}}(\alpha) \stackrel{\text { def }}{=} \sum_{n \sim N} f(n) e(n \alpha)
$$

where $n \sim N$ means that $n \in \mathcal{N} \stackrel{\text { def }}{=}(N, 2 N] \cap \mathbb{N}$ and $e(\alpha) \stackrel{\text { def }}{=} e^{2 \pi i \alpha}$, as usual. If $f$ is the convolution product of $g$ and the constantly 1 function, i.e.

$$
f(n)=(g * \mathbf{1})(n)=\sum_{d \mid n} g(d),
$$

we say, with Wintner [W], that $g=f * \mu$ is the Eratosthenes transform of $f$ (where $\mu$ is the well-known Möbius function). We call $f$ a sieve function of range $Q$ if its Eratosthenes transform $g$ is essentially bounded, namely $g(d) \ll_{\varepsilon} d^{\varepsilon}, \forall \varepsilon>0$, and vanishes outside $[1, Q]$ for some $Q \in \mathbb{N}$, that is to say,

$$
f(n)=\sum_{\substack{d \mid n \\ d \leq Q}} g(d) .
$$

Note that $f=g * \mathbf{1}$ is essentially bounded if and only if so is $g$.

As usual, $\ll$ is Vinogradov's notation, synonymous to Landau's $O$-notation. In particular, $\ll_{\varepsilon}$ means that the implicit constant might depend on an arbitrarily small and positive real number $\varepsilon$, which might change at each occurrence. We also write $g_{Q} \stackrel{\text { def }}{=} g \cdot \mathbf{1}_{Q}$ to mean that $g$ vanishes outside $[1, Q]$, the function $\mathbf{1}_{Q}$ being the indicator of $[1, Q] \cap \mathbb{N}$. In the above notation, we have set $f_{\mathcal{N}} \stackrel{\text { def }}{=} f_{2 N}-f_{N}$.

In [CL2] we have established a formula that relates the so-called $\ell$ th Ramanujan coefficient of a real sieve function $f$ of range $Q$, i.e.

$$
R_{\ell}(f) \stackrel{\text { def }}{=} \sum_{d \equiv 0(\ell)} \frac{g_{Q}(d)}{d}
$$

to the values of $\widehat{f_{\mathcal{N}}}$ attained at rational numbers; hereafter we write $n \equiv a(q)$ to abbreviate $n \equiv a(\bmod q)$. More precisely, Lemma 3.1 [CL2] states that

$$
\widehat{f_{\mathcal{N}}}(j / \ell)=R_{\ell}(f) N+O_{\varepsilon}\left((\ell Q)^{\varepsilon}(Q+\ell)\right), \quad \forall \ell>1, \forall j \in \mathbb{Z}_{\ell}^{*},
$$

which holds uniformly in the complete set $\mathbb{Z}_{\ell}^{*}$ of reduced residue classes modulo $\ell$. Moreover, it easily seen that (compare also $[\mathrm{C}]$ )

$$
R_{\ell}(f)=\frac{1}{\ell} \sum_{m \leq Q / \ell} \frac{g(\ell m)}{m} \ll_{\varepsilon} \frac{Q^{\varepsilon}}{\ell}
$$

Math Subject Classification 2010 : 11N37 Keywords: arithmetic progressions, short intervals 
Here we continue our study of the distribution of a real sieve funtion $f$ over short arithmetic bands, i.e.

$$
\bigcup_{1 \leq a \leq H}\{n \in(N, 2 N]: n \equiv a(\bmod q)\} \text {, with } H=o(N) .
$$

Let us recall that in [CL2] we have proved that the inequality (hereafter, we omit $a \geq 1$ in sums like $\sum_{a \leq H}$ )

$$
T_{f}(q, N, H) \stackrel{\text { def }}{=} \sum_{a \leq H} \sum_{\substack{n \sim N \\ n \equiv a(q)}} f(n)-\frac{H}{q} \widehat{f_{\mathcal{N}}}(0) \ll_{\varepsilon} N^{\varepsilon}(N / q+q+Q)
$$

holds for every real sieve function $f$ of range $Q \ll N$, after assuming that $H=o(q)$, as $q \rightarrow \infty$, and $q=o(N)$, as $N \rightarrow \infty$. Such conditions are required in order to avoid overlapping and sporadicity of the arithmetic bands, respectively. By a straightforward application of (1) and (2) here we generalize the previous inequality for

$$
T_{f}(q, r, b, N, H) \stackrel{\text { def }}{=} \sum_{a \leq H} \sum_{\substack{n \sim N \\ n \equiv a r+b(q)}} f(n)-\frac{H}{q} \widehat{f_{\mathcal{N}}}(0),
$$

where $r, b \in \mathbb{Z}$ are such that $(r, q)=1$ (hereafter $(r, q)=$ g.c.d. $(r, q)$, as usual). In particular, note that $T_{f}(q, 1,0, N, H)=T_{f}(q, N, H)$.

Theorem. Let $q, N, H, Q \in \mathbb{N}$ be such that $Q \ll N, H=o(q)$ and $q=o(N)$. For every sieve function $f: \mathbb{N} \rightarrow \mathbb{R}$ of range $Q$ and all $r, b \in \mathbb{Z}$ such that $(r, q)=1$, one has

$$
T_{f}(q, r, b, N, H) \ll_{\varepsilon} N^{\varepsilon}(N / q+q+Q) .
$$

Proof. By the orthogonality of additive characters $e_{q}(t) \stackrel{\text { def }}{=} e(t / q),(q \in \mathbb{N}, t \in \mathbb{Z})$, we get

$$
\begin{gathered}
T_{f}(q, r, b, N, H)=\frac{1}{q} \sum_{a \leq H} \sum_{n \sim N} f(n) \sum_{k \leq q} e_{q}(k(a r+b-n))-\frac{H}{q} \widehat{f_{\mathcal{N}}}(0)=\frac{1}{q} \sum_{k<q} \sum_{a \leq H} e_{q}(k(a r+b)) \widehat{f_{\mathcal{N}}}(-k / q) \\
=\frac{1}{q} \sum_{\substack{\ell \mid q \\
\ell>1}} \sum_{j \in \mathbb{Z}_{\ell}^{*}} \widehat{f_{\mathcal{N}}}(-j / \ell) \sum_{a \leq H} e_{\ell}(j(a r+b)),
\end{gathered}
$$

where we have set $\ell=q /(k, q)$ and $j=k /(k, q)$. Since $(r, q)=1$, for any $\ell \mid q$ there exists an integer $\bar{r}$ such that $r \bar{r} \equiv 1(\bmod \ell)$. Therefore we write

$$
T_{f}(q, r, b, N, H)=\frac{1}{q} \sum_{\substack{\ell \mid q \\ \ell>1}} \sum_{j \in \mathbb{Z}_{\ell}^{*}} \widehat{f_{\mathcal{N}}}\left(-\frac{j \bar{r}}{\ell}\right) e_{\ell}(j \bar{r} b) \sum_{a \leq H} e_{\ell}(j a) .
$$

By applying (1), (2) and the well-known inequality (see [Da], Ch.25)

$$
\sum_{V_{1}<v \leq V_{2}} e(v \alpha) \ll \min \left(V_{2}-V_{1}, \frac{1}{\|\alpha\|}\right)
$$

we conclude that

$$
\begin{gathered}
T_{f}(q, r, b, N, H) \ll_{\varepsilon} \frac{1}{q} \sum_{\ell \mid q, \ell>1}\left(\left|R_{\ell}(f)\right| N+(\ell Q)^{\varepsilon}(Q+\ell)\right) \sum_{j \in \mathbb{Z}_{\ell}^{*}} \frac{1}{\|j / \ell\|} \\
\ll_{\varepsilon} \frac{N^{\varepsilon}}{q} \sum_{\ell \mid q, \ell>1}\left(\frac{N}{\ell}+Q+\ell\right) \ell \ll_{\varepsilon} N^{\varepsilon}\left(\frac{N}{q}+Q+q\right),
\end{gathered}
$$

that is $(4)$. 
Remark 1. Besides (4), from the previous proof it transpires that also the upper bound of

$$
T_{f}(q, r, b, N, H)=\frac{1}{q} \sum_{\substack{\ell \mid q \\ \ell>1}} \sum_{j \in \mathbb{Z}_{\ell}^{*}} \widehat{f_{\mathcal{N}}}\left(-\frac{j \bar{r}}{\ell}\right) e_{\ell}(j \bar{r} b) \sum_{a \leq H} e_{\ell}(j a) \ll \frac{1}{q} \sum_{\substack{\ell \mid q \\ \ell>1}} \ell \sum_{\substack{j \leq \ell / 2 \\(j, \ell)=1}} \frac{1}{j} \max _{j \in \mathbb{Z}_{\ell}^{*}}\left|\widehat{f_{\mathcal{N}}}(j / \ell)\right|
$$

is independent of both $b$ and $r$ such that $(r, q)=1$ (that is to say, it is the same upper bound obtained for $r=1$ and $b=0)$.

Remark 2. Recalling that here trivial bound means $\ll N^{1+\varepsilon} H / q$, both (3) and (4) are non-trivial once the width $\theta \stackrel{\text { def }}{=} \frac{\log H}{\log N}$ is positive, $q \ll \sqrt{N^{1-\delta} H}$ and $q Q \ll N^{1-\delta} H$, for a suitable $\delta>0$. Since it is assumed that $Q \ll q \ll Q$ hereafter, we get a bound $Q \ll \sqrt{N^{1-\delta} H}$ and thus go beyond the square-root of $N$ (for $\theta>0$ ). Consistently with the terminology introduced in [CL2], we stop at the barrier $\frac{\log Q}{\log N}<\frac{1+\theta}{2}$.

In the final section of [CL2] we compared our study of the distribution of sieve functions in arithmetic bands to the classical results on the distribution in arithmetic progressions, which deal mostly with the overcoming of the so-called level $1 / 2$. In fact, our present results, insofar they generalize our previous ones, already go beyond level $1 / 2$.

Here we discuss the possibility of going beyond $1 / 2+\theta / 2$ in the present contest of the sieve functions in arithmetic bands, namely by taking $Q>\sqrt{N^{1+\delta} H}$ for a certain small and fixed $\delta>0$, so that $N^{1+\varepsilon} H / Q=$ $o(Q)$, provided $\delta>\varepsilon$. Indeed, in section 3 we exhibit a particular sieve function whose behavior in arithmetic bands confirms the optimality of such level.

\section{Length inertia in arithmetic bands.}

In [CL1] we studied the so-called length inertia property for weighted Selberg integrals (see [CL2] for the link between such integrals and the distribution of a sieve function in arithmetic bands). Such a property permits transfer of non-trivial bounds in short intervals of length $h$, say, to similar bounds in longer intervals of length $H=\infty(h)$ (that is $h=o(H)$, as $H \rightarrow \infty$ ). Here we show that a length inertia property holds also for the distribution of a sieve function in arithmetic bands. Indeed, we have

$$
\begin{gathered}
T_{f}(q, N,[H / h] h) \stackrel{\text { def }}{=} \sum_{a \leq[H / h] h} \sum_{\substack{n \sim N \\
n \equiv a(q)}} f(n)-\frac{[H / h] h}{q} \widehat{f_{\mathcal{N}}}(0)=\sum_{j \leq[H / h]}\left(\sum_{(j-1) h<a \leq j h} \sum_{\substack{n \sim N \\
n \equiv a(q)}} f(n)-\frac{h}{q} \widehat{f_{\mathcal{N}}}(0)\right) \\
=\sum_{j \leq[H / h]}\left(\sum_{a \leq h} \sum_{\substack{n \sim N \\
n \equiv a+(j-1) h(q)}} f(n)-\frac{h}{q} \widehat{f_{\mathcal{N}}}(0)\right)=\sum_{j \leq[H / h]} T_{f}(q, 1,(j-1) h, N, h) .
\end{gathered}
$$

\section{Optimality of the level.}

Let us set $\operatorname{sgn}(x) \stackrel{\text { def }}{=} x /|x|$ for all $x \in \mathbb{R} \backslash\{0\}$ and $\operatorname{sgn}(0) \stackrel{\text { def }}{=} 0$. Then, for any fixed $q \in \mathbb{N} \cap(Q, 2 Q]$, we define the arithmetic function $g$ as

$$
g(d)=g(d, q, N, H) \stackrel{\text { def }}{=} \operatorname{sgn}\left(\sum_{a \leq H}\left(\sum_{\substack{m \sim N / d \\ m d \equiv a(q)}} 1-\frac{1}{q} \sum_{m \sim N / d} 1\right)\right),
$$

if $d \in \mathbb{N} \cap(Q, 2 Q]$ and $g(d) \stackrel{\text { def }}{=} 0$ otherwise. It is plain that $g$ is the Eratosthenes transform of the sieve function $f(n)=f(n, q, N, H) \stackrel{\text { def }}{=} \sum_{d \mid n} g(d)$ of range $2 Q$. By noting that $\operatorname{sgn}(x) x=|x|$ for all $x \in \mathbb{R}$, we write

$$
\left|T_{f}(q, N, H)\right|=\left|\sum_{a \leq H}\left(\sum_{\substack{n \sim N \\ n \equiv a(q)}} f(n)-\frac{1}{q} \sum_{n \sim N} f(n)\right)\right|=\left|\sum_{d \sim Q} g(d) \sum_{a \leq H}\left(\sum_{\substack{m \sim N / d \\ m d \equiv(q)}} 1-\frac{1}{q} \sum_{\substack{m \sim N / d \\ m \sim N}} 1\right)\right|
$$




$$
=\sum_{d \sim Q}\left|\sum_{a \leq H}\left(\sum_{\substack{m \sim N / d \\ m d \equiv a(q)}} 1-\frac{1}{q} \sum_{m \sim N / d} 1\right)\right| .
$$

In order to show that $\left|T_{f}(q, N, H)\right| \gg N H / q$, we set

$$
\begin{aligned}
\mathcal{S}=\mathcal{S}(q, Q, H, N) & \stackrel{\text { def }}{=}\left\{d \in \mathbb{N} \cap(Q, 2 Q]: \sum_{a \leq H} \sum_{\substack{m \sim N / d \\
m d \equiv a(q)}} 1 \geq 1\right\}, \\
& \stackrel{\mathcal{d e f}}{=}(\mathbb{N} \cap(Q, 2 Q]) \backslash \mathcal{S}
\end{aligned}
$$

and prove that $|\mathcal{S}|=o(Q)$, which in turns yields $|\mathcal{E}| \gg Q$. Indeed, from the latter inequality we observe that

$$
\begin{gathered}
\left|T_{f}(q, N, H)\right|=\sum_{d \in \mathcal{S}}\left|\sum_{a \leq H}\left(\sum_{\substack{m \sim N / d \\
m d \equiv a(q)}} 1-\frac{1}{q} \sum_{m \sim N / d} 1\right)\right|+\sum_{d \in \mathcal{E}} \frac{H}{q} \sum_{m \sim N / d} 1 \\
\gg|\mathcal{E}| \frac{N H}{q Q} \gg \frac{N H}{q} .
\end{gathered}
$$

Now let us prove that $|\mathcal{S}|=o(Q)$. To this end, after recalling that the divisor function $\mathbf{d}(n) \stackrel{\text { def }}{=} \sum_{d \mid n} 1$ is essentially bounded and $q>Q$, we note that

$$
|\mathcal{S}| \leq \sum_{d \in \mathcal{S}_{q}} \sum_{a \leq H} \sum_{\substack{m \sim N / d \\ m d \equiv a(q)}} 1 \leq \sum_{a \leq H} \sum_{\substack{n \sim N \\ n \equiv a(q)}} \mathbf{d}(n) \ll_{\varepsilon} \frac{N^{1+\varepsilon} H}{Q} .
$$

If $Q>\sqrt{N^{1+\delta} H}$ for a certain $\delta>0$, then $N^{1+\varepsilon} H / Q=o(Q)$ once $\varepsilon<\delta$, that yields the desired conclusion.

\section{Concluding remarks.}

Sieve functions are ubiquitous in analytic number theory. Besides the truncated divisor sum $\Lambda_{R}$ (see [G]), that is a linear combination of sieve functions of range $R$ (see [CL2] for our remarks on $\Lambda_{R}$ ), we quote the so-called restricted divisor function

$$
\tau_{Q}(n) \stackrel{\text { def }}{=}\left(\mathbf{1}_{Q} * \mathbf{1}\right)(n)=\sum_{d \mid n, d \leq Q} 1
$$

whose Eratosthenes transform is the indicator $\mathbf{1}_{Q}$ of $[1, Q] \cap \mathbb{N}$. We refer the reader to $[\mathrm{T}]$ for results on the distribution of $\tau_{Q}$ in short arithmetic progressions. Here we wish to stress that in $[\mathrm{T}]$ one finds also conjectures and average results concerning the distribution in arithmetic bands of the more complicated function

$$
\tau_{Q, R}(n) \stackrel{\text { def }}{=}\left(\mathbf{1}_{Q} * \mathbf{1}_{R}\right)(n)=\sum_{\substack{(d, t) \\ d t=n}} \mathbf{1}_{Q}(d) \mathbf{1}_{R}(t) .
$$

Such an essentially bounded function is linked to the pair correlation problem for fractional parts of the quadratic function $\alpha k^{2}$, with $k \in \mathbb{N}$ and $\alpha \in \mathbb{R}$ (compare also [S]). While in [T], Conjecture 1.2, it is pursued the research of an upper bound for

$$
\sum_{a \leq H} \sum_{n \equiv a r(q)} \tau_{Q, R}(n)-\frac{H Q R}{q}, \text { with }(r, q)=1,
$$

under suitable conditions on $H, Q, R$, here our Theorem leads to an asymptotic formula for the inverse Eratosthenes transform of $\tau_{Q, R}$ in arithmetic bands, namely for

$$
\sum_{a \leq H} \sum_{n \equiv a r(q)}\left(\tau_{Q, R} * \mu\right)(n) .
$$


In [CL3] we established an asymptotic inequality for the exponential sum associated to the localized divisor functions, a family of functions including the aforementioned $\tau_{Q}, \tau_{Q, R}$, and the standard divisor function $d_{k}$, $k \geq 2$ (recall that $d_{k}(n)$ is the number of ways to write $n$ as a product of $k$ positive integers). The particular instance of such an inequality for $d_{k}$ is

$$
\sum_{n \sim N} d_{k}(n) e(n \alpha) \ll_{k, \varepsilon}(N q)^{\varepsilon}\left(N / q+q+N^{1-1 / k}\right),
$$

for all $\alpha \in\left[a / q-1 / q^{2}, a / q+1 / q^{2}\right]$, with $(a, q)=1, q>1$. Somehow, this can be regarded as being analogous to the inequality which follows by combining (1) with (2). Such a circumstance is remarkable even in view of the fact that any $d_{k}$ falls short of being a sieve function, with a sort of Eratosthenes transform which turns out to be the sum of $d_{k-1}$ plus some restricted divisor functions (see the last section of [CL1]).

Finally, since the function proposed in section 3 seems to be rather artificial in that it depends on a fixed modulus $q \in \mathbb{N} \cap(Q, 2 Q]$, an intriguing open question to ask is how many standard sieve functions might support the optimality of the level accomplished by our Theorem and the results of [CL2].

\section{References}

[C] G. Coppola, On some lower bounds of some symmetry integrals, Afr. Mat. 25, issue 1 (2014), 183-195. MR : 3165958

[CL1] G. Coppola and M. Laporta, Symmetry and short interval mean-squares, (2016), arXiv: 1312.5701 (submitted)

[CL2] G. Coppola and M. Laporta, Sieve functions in arithmetic bands, Hardy-Ramanujan J. 39, (2016), 21-37

[CL3] G. Coppola and M. Laporta, A note on the exponential sums of the localized divisor functions, to appear in PALANGA 2016 Conference Proceedings

[Da] H. Davenport, Multiplicative Number Theory. 3rd edition, GTM 74, Springer, New York, 2000.

[G] D.A. Goldston, On Bombieri and Davenport's theorem concerning small gaps between primes, Mathematika, 39 (1992), 10-17

[S] I.E. Shparlinski, On the restricted divisor function in arithmetic progressions, Rev. Mat. Iberoam., 28, (2012), 231-238

[T] J.L. Truelsen, Divisor problems and the pair correlation for the fractional parts of $n^{2} \alpha$, Int. Math. Res. Not. IMRN, 2010 (2010), 3144-3183

[W] A. Wintner, Eratosthenian Averages, Waverly Press, Baltimore, MD, 1943

Giovanni Coppola

Home address : Via Partenio 12

83100, Avellino, ITALY

e-page: www.giovannicoppola.name

e-mail: giocop@interfree.it

Maurizio Laporta

Università degli Studi di Napoli "Federico II",

Dipartimento di Matematica e Applicazioni "R.Caccioppoli",

Complesso di Monte S.Angelo, Via Cinthia - 80126, Napoli, ITALY

e-mail: mlaporta@unina.it 